\title{
An U pdate Review of Animal Models For Experimental Periodontal Diseases
}

\author{
Mounika Basavaraju1, Vidya Sagar Sisinity², \\ Rajababu Palaparthi ${ }^{3}$, Pavan Kumar Addanki ${ }^{4}$
}

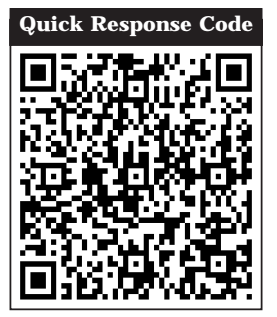

doi: $10.5866 / 2015.7 .10268$

${ }^{1 \& 4}$ Post Graduate Student

${ }^{2}$ Professor

${ }^{3}$ Professor and HOD

Department of Periodontics

Kamineni Institute of Dental Sciences

Narketpally, Nalgonda Dist. India

\section{Article Info:}

Received: October 13, 2015

Review Completed: November 12, 2015

Accepted: December 11, 2015

Available Online: March, 2016 (www.nacd.in)

(C) NAD, 2015 - All rights reserved

\section{E mail for correspondence:}

mounika.kaushik@gmail.com

\begin{abstract}
:
Animal models and cell cultures have contributed new knowledge in biological sciences, including Periodontology. Although cultured cells can be used to study physiological processes that occur during the pathogenesis of periodontitis, the complex host response fundamentally responsible for this disease cannot be reproduced in vitro. Among the animal kingdom, rodents, rabbits, pigs, dogs, and nonhuman primates have been used to model human periodontitis, each with advantages and disadvantages. Periodontitis commonly has been induced by placing a bacterial plaque retentive ligature in the gingival sulcus around the molar teeth. In addition, alveolar bone loss has been induced by inoculation or injection of human oral bacteria (e.g., Porphyromonas gingivalis) in different animal models. A practical and highly reproducible model that truly mimics the natural pathogenesis of human periodontal disease has yet to be developed.
\end{abstract}

Key words: Periodontal disease, animal models, biomaterials, periodontal surgery.

\section{INTRODUCTION:}

Periodontitis is a highly prevalent, chronic immuno inflammatory disease of the periodontium that results in progressive loss of gingival tissue, the periodontal ligament, and adjacent supporting alveolar bone. ${ }^{1}$ Chronic inflammation of the periodontium is initiated by complex subgingival biofilms containing several likely periodontal pathogens. As individuals are not equally susceptible to the destructive effects of periodontal infections, periodontitis is not only caused by bacterial infection but also may be associated with host susceptibility. ${ }^{2}$ Variability in host responses among individuals contributes significantly to the expression of periodontal diseases. Although human cell cultures were found to be useful models for replicating some aspects of the periodontal disease process at the cellular level, information about the complex host response was not prominent. ${ }^{3}$ Thus, research into the host response using animals is 
critically important in the analysis of periodontal disease and development of improved treatments. Animal models have contributed to the generation of new knowledge in biological sciences, including Periodontology. Various species have been used to study the pathogenesis of periodontitis and to assess therapeutic modalities against the disease. The present review focuses on naturally and experimentally induced defects in animal models which are used to study different aspects of periodontal diseases.

\section{Need for Animal Models:}

Animal research and its value to human experience remain controversial. Animal data can provide us with models of biologic trends before proceeding to human application. Animal models have been used to evaluate the pathogenesis of periodontal diseases and various periodontal treatment modalities. Human longitudinal studies of periodontal diseases pose many problems such as determining the level of disease activity, individuals at risk and susceptibility to disease progression. It is important to choose an animal model that has similar characteristics of human anatomy and periodontal diseases. F eatures of periodontal diseases in human and animals vary greatly depending upon which form of the disease is present and the stage of the development. In relatively new era of periodontics like regenerative procedures, bone grafts and implant surgical procedures have shown promising results in animal studies. Theresults obtained may serve to stimulate further efforts and promote further steps in the right direction. ${ }^{4}$

\section{Selection of Model:}

The selection of an experimental model is determined by research objectives, as well as laboratory constraints such as housing of large or non standard animals. I n most cases, small animal models such as rats or hamsters will be sufficient to assess the role of bacteria, diet or

other factors in periodontal inflammation at the histological level, providing sufficient statistical significance and pre-clinical relevance. The use of large animals with ethical and social issues such as monkeys and dogs should be reserved for last phase validation of new treatments prior to use in human clinical practice. The selection of these animals was based on similar pathologies and the ease of surgically created clinically relevant defects. Experimental periodontal defects may be obtained in three different ways: ${ }^{5}$

1. The acute defect model

2. The chronic defect model

3. The acute/chronic defect model.

In the acute model, all defects are surgicallyinduced by removing surgically all the periodontal components (bone, cementum and periodontal ligament). Reproducibledefects in experimental and control sites are created. In the chronic model, lesions are obtained by placing orthodontic elastics, silk sutures or ligatures around teeth during 12 to 20 weeks, depending on the type of animal studied. These defects are deeper in the interproximal spaces than in the buccal or lingual surfaces. In the combined acute/chronic model, the defects are surgically-created and ligatures are placed to ensure calculus accumulation and to prevent spontaneous regeneration of the defects.

\section{Various Animal Models in Periodontology:}

The animals used in periodontal research have a different anatomy, dentition and structure of periodontal tissues. Furthermore, the physiopathology, oral bacteria and inflammation response vary between species.

\section{Non-human primates:}

Monkeys have the advantage of probably being phylogenetically similar to humans. All these species are diphyodont. Macaques, baboons and chimpanzees have the same dental formula as humans. However, marmosets have a different formula. The anatomy of teeth and roots is close to that of humans, but the size is smaller. The canines in most non-human primates are elongate and prehensile. In gorillas and baboons, the premolars have more than one root. Histologically, the structure of the periodontium is also similar to that observed in humans. Microbiologically, in Macaca fascicularis (cynomol gus monkeys), the composition of the plaque is Gram positive rods and cocci for supragingival plaque and anerobic Gram negative rods for subgingival plaque.6,7 The inflammatory response to periodontal disease is quite similar to that found in humans. Connective tissues are infiltrated by plasma cells, Iymphocytes and neutrophils. In other species, such as squirrel monkeys and marmosets, there is very limited 


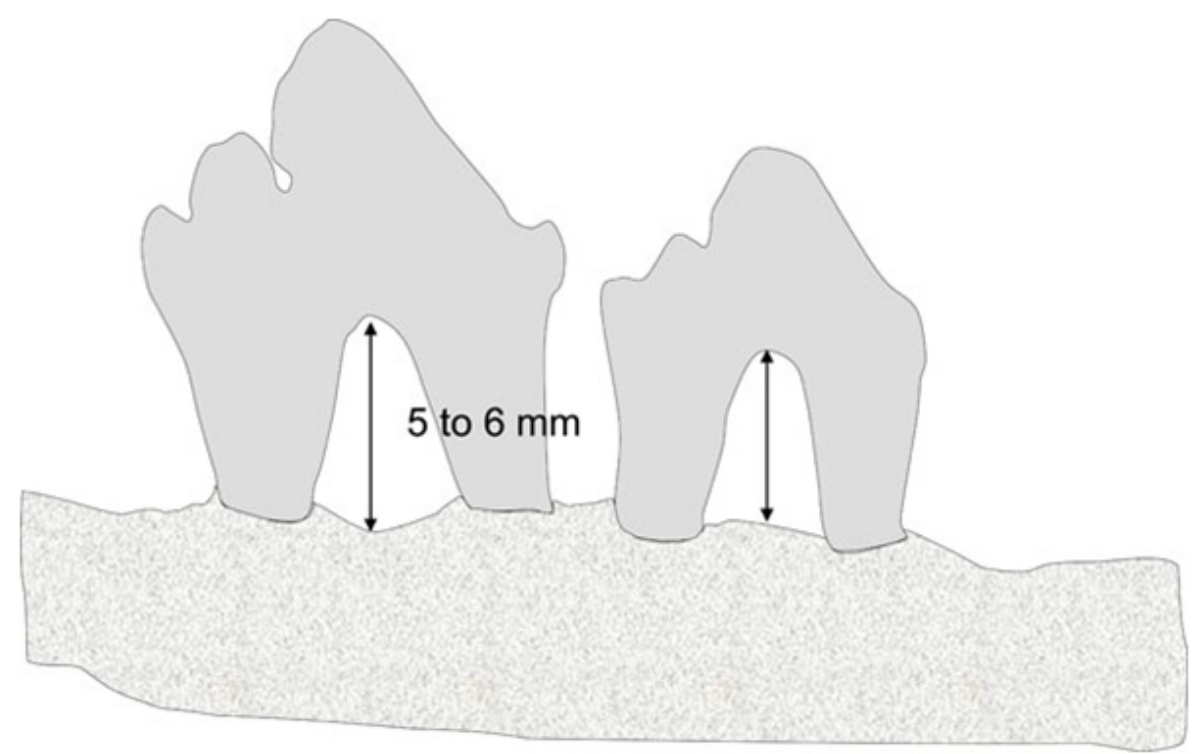

Figure 1: Critical sized supra alveolar defect model in Dogs given by Wikesjo(1994).

inflammatory infiltrate. These major differences with humans make them inappropriate models for studying the pathogenesis of periodontitis. ${ }^{8}$

Monkeys were used because they present anatomical similarities with humans. However, it is difficult to obtain a large number of animals and it is thus imperative that a maximum number of defects be created in order to obtain a sufficient quantity of test and control sites to make adequate statistical analysis of the results possible. Moreover, ethical considerations and regulations should be fulfilled in order to prevent any trafficking of protected species. ${ }^{6}$

\section{Dogs:}

Many experimental studies on gingival and periodontal diseases have been conducted in dogs. The beagle is one of the most commonly used due to its size and its extremely cooperative temperament. Globally, all periodontal tissues and the size of the teeth are quite similar to those observed. However, some major differences exist between dogs and humans as thelack of lateral movements, no occlusal contacts for all the premolars and presence of open contacts between teeth. The frequent lack of gingival sulci and crevicular fluid, a different composition of periodontal plaque and calculus are other important differences between dogs and humans. ${ }^{9}$ All dogs are diphyodont with deciduous and permanent dentition. All domestic dogs have a natural susceptibility to periodontal diseases in adult age but may be maintained healthy by appropriate plaque control. Periodontal alterations, including gingivitis and periodontitis, increase in prevalence and severity with age, faster than in man but with the same etiologic factors. Gingivitis may be generalized and associated with bleeding on probing, the presence of supragingival and subgingival plaque, calculus and severe bone loss including osseous defects.

Currently, gingival recessions appear in the severe forms of periodontal diseases in dogs. Supragingival plaque is mostly composed of Gram positive cocci. The subgingival flora is mainly anaerobic Gram negative cocci and rods. In healthy dogs, the gingival sulcus is most frequently absent. Thejunctional epithelium and epithelial attachment extend to the most coronal level on the gingival margin. In early gingivitis, the inflammation is limited to this marginal part of the gingival tissue with the presence of neutrophils and monocytes leaving most of the connective tissue free of infiltrate. At the later stages, the infiltrate extends apically subjacent to the junctional epithelium with the formation of gingival pockets. There is a formation of periodontal pockets lined with typical pocket epithelium.

In the connective tissue, the dense cellular infiltrate consists mainly of plasma cells and lymphocytes. The osteoclastic resorption of alveolar bone may result in deep, narrow lesions extending 
vertically around a single root leaving the interdental space. The differences can be explained more by the nature of the infection or genetics than by diet. In these natural periodontal diseases, the extent and localizations of the periodontal lesions are not homogeneous, which may be considered to be a limitation for the model. A surgical model was proposed by Wikesjo in 1994. Critical-sized supraalveolar defects measuring $6 \mathrm{~mm}$, were created at the level of the mandibular premolars (F igure 1$).{ }^{10}$,

${ }^{11}$ The class III furcation defects thus obtained made it possible to test various regeneration procedures, whether or not they were associated with a filling biomaterial.

\section{Rats:}

The rat is the most extensively-studied rodent for the pathogenesis of periodontal diseases. The structure of the dental gingival area in rats is quite similar to that observed in humans, ${ }^{12}$ with a shallow gingival sulcus and attachment of the junctional epithelium to the tooth surface. The most commonlyused strains are Wistar or the Spraque-Dawley. In rats, periodontitis appears to be an infectious process. I noculations or injections of various periodontal pathogens such as Prophyromonas gingivalis, Aggregatibacter acti nomycetemcomitans, Fusobacterium nucleatum, Capnocytophaga, Eikenella corrodens, Actinomyces viscosus, and Streptoccocus sobrinus can induce periodontal lesions. Furthermore, rats are easy to handle.

\section{Hamsters:}

In hamsters, periodontal disease does not occur spontaneously but may be obtained experimentally. The golden Syrian hamster is the most commonly used. Histologically, the structure of the periodontal tissue is very similar to that of rats but due to the small size of this kind of animal, the interdental septum is narrower than in rats. Spontaneous periodontal disease was obtained using an appropriate diet containing high concentrations of carbohydrates, particularly sucrose. ${ }^{13}$ In summary, as in rats, the inflammatory response is very limited in hamsters and is very different from that observed in humans. The mechanisms of alveolar bone resorption in hamsters with diet dependent periodontal lesions are quite similar to those observed in rats infected with Gram positive bacteria.

\section{Ferrets:}

Ferrets have a deciduous and permanent dentition. Ferrets (Mustela putorius) naturally develop calculus and periodontal disease similar to humans. ${ }^{14}$ Unlike rodents, calculus formation in ferrets does not depend on the diet and can bescored in live ferrets. Ferrets are a suitable model to study calculus, however, they can easily escape from standard cages and they need special maintenance.

\section{Minks:}

In minks, spontaneous periodontitis, which is age- and plaque-dependent, is observed. Nevertheless, the extent of this periodontal disease appears to be severe only in very old animals. In minks, neutrophils play a key role in periodontal destruction due to deficiencies in the chemotactic response and massive release of Iysosomal enzymes and proteases into periodontal tissue. Minks are therefore interesting experimental models in research on the etiology of periodontal diseases. Nevertheless, housing these animals may be difficult or require specific authorizations that may explain theabsence of recent publications in the literature. ${ }^{15}$

\section{Rabbits:}

Characterization of the oral microorganisms in rabbits showed numerous pathogenic bacteria, including $\mathrm{F}$. nucleatum, Prevotella spp., P. micros, S. milleri group, A. israelii, and A. haemolyticum, which is somehow consistent with the flora related to periodontal disease in humans. Rabbits have been used for creation of surgically induced periodontal defect and to study periodontal regeneration, but they have been found less suitable for regeneration of periodontal ligament. ${ }^{16}$

\section{Other Animals}

Other animal models have been investigated for modeling periodontal diseases.

Horses: Common naturally occurring oral diseases in horses include buccal abrasions, calculus, gingival recession, and periodontal pockets. According a recent equine survey, the prevalence of periodontal pockets and gingival recession is highest in older horses and mostly associated with other dental disorders and tooth loss. ${ }^{17}$ Because of their size and husbandry considerations, horses are not a practical model for basic science studies of periodontitis or for testing of potential therapies. 
Sheep: They have also been studied in the context of periodontal diseases. ${ }^{18}$

Miniature pigs: The miniature pig model has emerged as a good alternative to the dog model. These animals are similar to humans in gross anatomy and physiology and have economic advantages over other large species. The periodontium of miniature pigs shows many similarities to that of humans. The depth of the gingival sulcus is $2-3 \mathrm{~mm}$ with a long junctional epithelium. Natural gingivitis can be observed at 6 months of age. Almost the earliest non disease use of miniature pigs in periodontal research was for the creation of mucogingival defects. Miniature pigs have al so been tested for suitability in experimental peri-implantitis studies. ${ }^{19}$

Cats: One publication used the cat as animal model. In this research, class III furcation defects were surgically created at the level of the premolars in order to study ankylosis at root level during periodontal healing. ${ }^{20}$

\section{Conclusion:}

Experimental models for periodontal diseases are essential for understanding the origin and evolution of the pathology in humans. The use of animal models in periodontal research is a necessary step prior to entering into clinical trials with new biomaterials and treatments. A more systematic use of these small animal models appears evident for future research, especially from a surgical point of view.

\section{REFERENCES}

1. Pihlstrom BL, Michalowicz BS, J ohnson NW. Periodontal diseases. Lancet 2005; 366:1809-1820.

2. Oz HS, Puleo DA .Animal models for periodontal diseases. J Biomed Biotechnol 2011:1-8.

3. Schenkein HA. Host responses in maintaining periodontal health and determining periodontal disease. Periodontol 2000 2006; 40:77-93.

4. Weinberg MA, Bral M. Laboratory animals models in periodontology. J Clin periodontol 1999; 26:335-340

5. Caton J, Mota L, Gandini L, Laskaris B. Non-human primate models for testing the efficacy and safety of periodontal regeneration procedures. J Periodontol 1994; 65:1143-1150.
6. GiannobileWV, Finkelman RD, and Lynch SE. Comparison of canine and non-human primate animal models for periodontal regenerative therapy: results following a single administration of PDGF/IGF-I.J Periodontol 1994; 65:11581168.

7. Socransky SS, Haffajee AD. The bacterial etiology of destructive periodontal disease: current concepts. J Periodontol 1992; 63: 322-331.

8. Page R, Schroeder H. Periodontitis in man and other animals. A comparative review. Basel: Karger 1982.

9. Sorensen WP, Loe H, Ramfjord SP. Periodontal disease in the beagle dog: a cross sectional clinical study.J Periodontal Res 1980; 15:380-389.

10. Haney J M, Zimmerman GJ , Wikesjo UM. Periodontal repair in dogs: evaluation of the natural disease model. J Clin Periodontol 1995; 22:208-213.

11. Wikesjo UM, Kean CJ , Zimmerman GJ . Periodontal repair in dogs: supraal veolar defect models for evaluation of safety and efficacy of periodontal reconstructive therapy. J Periodontol 1994; 65:1151-1157

12. Yamasaki A, Nikai H, Niitani K, Ijuhin N. Ultrastructure of the junctional epithelium of germfree rat gingiva. J Periodontol 1979; 50:641-648.

13. Lallam-Laroye C, Escartin Q, Zlowodzki AS, et al. Periodontitis destructions are restored by synthetic glycosaminoglycan mimetic. J Biomed Mater Res A 2006; 79: 675-683.

14. King J and Gimson A. Experimental investigations of periodontal disease in the ferret and related lesions in man. Br Dent J 1947; 83:126-127

15. Xavier Struillou, Hervé Boutigny, Assem Soueidan, and Pierre Layrolle. Experimental Animal Models in Periodontology: A Review. Open Dent J 2010; 4:37-47.

16. Tyrrell KL, Citron DM, J enkins J R et al. Periodontal bacteria in rabbit mandibular and maxillary abscesses. J Clin Microbiol 2002; 40:1044-1047.

17. Anthony J, Waldner C, Grier C, and Laycock AR. A survey of equine oral pathology J Vet Dent 2010; 27:12-15.

18. Cutress TW. Histopathology of periodontal disease in sheep. J Periodontol 1976; 47:643-650.

19. Kantarci A, Hasturk $\mathrm{H}$ and VanDyke J E Animal Models for Periodontal regeneration and peri implant responses. Periodontol 2000 2015; 68:66-82

20. Takahashi D, Odajima T, Morita M, Kawanami M, Kato H Formation and resolution of ankylosis under application of recombinant human bone morphogenetic protein-2 (rhBMP. 2) to class III furcation defects in cats. J Periodontal Res 2005; 40:299-305. 